\title{
Desplazamientos desde las pedagogías feministas: reflexiones posibles para un ejercicio docente situado
}

\author{
Displacements from feminist pedagogies: possible \\ reflections for a situated teaching exercise
}

\author{
Catalina Montenegro-GonZÁlez* \\ Universidad de Los Lagos \\ ORCID ID: 0000-0002-4432-5582 \\ Alejandra Paz CoRvalán-NAVIA* \\ Universidad Andrés Bello
}

ORCID ID: 0000-0002-6329-403X

Recibido: 23/06/2020

Aceptado: 02/09/2020

doi: https://doi.org/10.20318/femeris.2020.5760

Resumen. El presente artículo indaga y reflexiona sobre aspectos relevantes presentes en las pedagogías feministas, como forma de propiciar la transformación de espacios de aprendizaje, alejados del pensamiento hegemónico patriarcal. Estas reflexiones emergen desde los movimientos sociales de 2018 definidos por los medios de comunicación como mayo feminista en Chile. Comenzamos a hacernos preguntas sobre cómo los feminismos permean a la sociedad y a la pedagogía en un contexto latinoamericano-chileno y donde las estructuras sociales, culturales y políticas están siendo remecidas frente al cuestionamiento de una sistema educativo que no va en sintonía de las necesidades de la ciudadanía.

Con lo anterior, se toman algunas conceptualizaciones del pensamiento feminista latinoamericano y el feminismo de la diferencia sexual, para re-pensar los territorios educativos. Es así como este articulo indaga en las dinámicas del aprendizaje y se busca poner en valor aspectos relevantes para pensar las escuelas alejadas de prácticas discriminatorias, sexistas y marginadoras.

De esta forma emergen las ideas de experiencias como procesos de vivencia compartida con otras-os, los contextos diversos para pensar pedagogías significativas, las propias narrativas y la consideración de un aprendizaje colaborativo y de relaciones amorosas, siendo estas últimas una práctica constante en el quehacer de las movilizaciones feministas ocurridas en Chile durante mayo del 2018 y desde donde se abordan estos últimos planteamientos.

Este trabajo es una invitación a visualizar nuevas formas de aprendizaje, desde la consideración de la singularidad y las trayectorias vitales para la construcción del conocimiento.

Palabras clave: pedagogía feminista, contexto, experiencia, aprendizaje, docencia.

Abstract. The present article investigates and reflects on relevant aspects present in feminist pedagogies, as a way of propitiating the transformation of learning spaces, far from patriarchal hegemonic thought. These reflections emerge from the social movements of 2018

\footnotetext{
"catalina.montenegro@gmail.com
}

*alejandracorvalan@gmail.com 
defined by the media as feminist May (in Chile. We begin to ask ourselves questions about how feminisms permeate society and pedagogy in a Latin American-Chilean context and where social, cultural and political structures are being shaken in the face of the questioning of an educational system that is not in tune with the needs of the citizenry.

In addition of above, some conceptualizations of Latin American feminist thought are taken and feminism of sexual difference to rethink educational territories. This is how this article investigates the dynamics of learning and seeks to value relevant aspects to think about schools away from discriminatory, sexist and marginalizing practices.

In this way, the ideas of experiences emerge as processes of shared living with others, the diverse contexts for thinking about meaningful pedagogies, the narratives themselves and the consideration of collaborative learning and love relationships, the latter being a constant practice in the work of the 2018 feminist mobilizations and from where these last approaches are board.

This work is an invitation to visualize new forms of learning, from the consideration of the singularity and the vital trajectories for the construction of knowledge.

Keywords: feminist pedagogy, context, experience, learning, teaching

\section{Introducción}

En Chile desde mayo de 2018 en adelante se desarrollaron diversos acontecimientos políticos, sociales y económicos que tuvieron como eje central desafiar la dominación masculina en diferentes estructuras de la sociedad (Reyes-Housholder y Roque, 2019). Tras las movilizaciones acontecidas en este periodo, se cuestionó enérgicamente la manera en que se distribuye el poder entre varones y mujeres en diversos espacios de la sociedad chilena. En este proceso tanto escuelas, como universidades fueron un punto de partida, en donde mujeres, estudiantes y trabajadoras de la educación, sacaron a la luz pública debates en torno a temas como, el carácter androcéntrico de las instituciones, el sexismo en la educación y la violencia de género (Undurraga y Simburgüer, 2018; Cea, Opazo, Devaud, Lamadrid, Retamal, Zagal, Gatica, Martínez, Kvyeh y Gonzalez, 2018).

A propósito de la descripción de este fisurado presente, el cual toma fuerza gracias al fuerte cuestionamiento por parte del estudiantado, particularmente mujeres, se posiciona en el debate y la agenda política, las bases para repensar las estructuras educativas, poniendo en cuestión las formas de aprender, relacionarse y reflexionar tanto en universidades, como escuelas del país (Cabello 2018; Palma, 2018; Reyes-Housholder y Roque, 2019; Ubilla, Pérez, Leibe, López, Arce-Riffo y Vera, 2019). Estas reflexiones sociales que levantan una crítica a lo que Martínez y Ramírez (2017) definen como "agentes de socialización (familias, instituciones educativas, medios de comunicación, etc.)" los cuales "crean y transmiten códigos culturales que sustentan un sistema organizado en estructuras opresoras y que asumimos como legítimas y propias (2017, p.82). Este proceso provocó un cuestionamiento a toda la estructura educativa, donde los establecimientos públicos de Chile se vieron remecidos, dado que la fuerza del cuestionamiento del espacio educativo por parte del estudiantado, sobre todo mujeres que comenzaron a poner en tensión maneras de aprender, relacionarse y reflexionar en la escuela y las universidades.

Desde aquí, algunas ideas centrales de los planteamientos feministas comenzaron a hacerse presente en los espacios educativos, tensionando prácticas patriarcales y posiciones de sujetas-os históricamente naturalizadas e invisibilizadas en aula (Azúa 2016; Cabello 
2018; Richard, 2018; Reyes-Housholder y Roque, 2019; Ubilla, Pérez, Leibe, López, ArceRiffo y Vera, 2019; Undurraga y Simburgüer, 2018), abriendo las discusiones a la consideración de los feminismos como poderosas opciones de cambio, "al buscar, defender y proponer la construcción de relaciones de género basadas en la equidad y generar alternativas de acción ante los mecanismos de reproducción de desigualdades" (Martínez, 2016, p.131).

En concordancia con este proceso, emergen preguntas e inquietudes que se abordan en este artículo como ¿Cuáles son los planteamientos de las pedagogías feministas? o ¿Para qué abordamos estas pedagogías y en un contexto latinoamericano?

Este artículo realiza una revisión de literatura desde la década de los noventa en adelante, relacionado con las pedagogías feministas y las pedagogías críticas vinculadas a los procesos de aprendizaje dentro de aulas escolares, articulando estas reflexiones con los acontecimientos sociales ocurridos en Chile desde mayo del 2018 en adelante. Se propone un marco que permita reflexionar en torno a aspectos de la práctica pedagógica basado en el trabajo desarrollado por Belausteguigoitia y Mingo (1999) en su libro "Géneros Prófugos"; de esta forma se relevan elementos centrales pertenecientes a las pedagogías feministas con el objetivo de establecer un diálogo entre elementos pedagógicos, movimientos sociales, demandas que emergen de las luchas feministas, y planteamientos teóricos pertinentes.

A partir de esta revisión, se establecen relaciones entre los feminismos latinoamericanos y las propuestas desde norteamérica y Europa, con el objetivo de poner en el centro de la reflexión las aportaciones realizadas a la configuración de las pedagogías feministas en Chile e invitando a recrear un entramado epistemológico y ontológico que cruce planteamientos feministas tanto latinoamericanos, europeos y norteamericanos, persiguiendo una reflexión personal y contextualizada a los propios territorios de aprendizaje, donde las pedagogías feministas no solo resisten y responden a un sistema social patriarcal, sino que además esbozan nuevas posibilidades en las formas de aprendizaje, y en construir un tejido social alejado de prácticas hegemónicas.

\section{La escuela como espacio de producción y reproducción de sujetas-os sociales}

Diversos trabajos desde la sociología de la educación, han permitido reflexionar en torno al espacio educativo en tanto sistema, institución cultural, espacio de producción y reproducción de prácticas sociales e instancia de constitución de sujeta-os sociales y su relación con distintos ámbitos del orden social (Bertarelli 2014; Jose y Shanuga, 2018; Bunn, Threadgold y Burke, 2019; Malvankar, 2019; Voyer, 2018).

A partir de las aportaciones de diferentes teóricas-os, es posible comprender la escuela como un espacio en donde se perpetúan los modelos culturales de la sociedad (Bernstein 1994; Bourdieu y Passeron, 2009).

Tal como refiere Pérez, Nogueroles y Méndez (2017) dado que tanto varones como mujeres, somos sujeta-os socialmente construidos y es al interior de la escuela donde los niños aprenden a hacer uso de sus privilegios de género, mientras las niñas continúan 
siendo socializadas para aceptar lo que Bourdieu (2000) denominó la 'dominación masculina'. Esta dominación que puede ser entendida como una forma particular de violencia simbólica, según el autor:

Se instituye a través de la adhesión que el dominado se siente obligado a conceder al dominador (por consiguiente a la dominación) cuando no dispone, para imaginarla o para imaginarse a sí mismo o, mejor dicho, para imaginar la relación que tiene con él, de otro instrumento de conocimiento que aquel que comparte con el dominador. (Bourdieu, 2000, p.51).

Es a través de aquella dimensión simbólica de la violencia, que la visión androcéntrica "se impone como neutra y no siente la necesidad de enunciarse en unos discursos capaces de legitimarla (...) funciona como una inmensa máquina simbólica que tiende a ratificar la dominación masculina" (Bourdieu, 2000, p.22).

De forma sutil y cotidiana a través de la interacción con otras personas, en la infancia se establecen posiciones y prácticas entre varones y mujeres ligadas al devenir tanto de la identidad individual, como de la identidad social. Tal como refiere Bernstein: "las relaciones de clase generan, distribuyen, reproducen y legitiman formas características de comunicación, que transmiten códigos dominantes y dominados, y esos códigos posicionan de forma diferenciada a los sujetos en el proceso de adquisición de los mismos" (Bernstein, 1994, p.25). De esta manera, a través de diferentes procesos de interacción social se transmite una determinada distribución de poder y de las categorías culturales dominantes (Graizer y Saurin, 2011). Esto también ocurre con la categoría sexo/género dentro de la escuela, la cual no sólo va a implicar hablar de cuerpos sexuados, sino además de las prácticas sociales y educativas vinculadas a dichos cuerpos, que les dan contexto y forma (Bertarelli, 2014).

Bourdieu (1991) y Bernstein (1994) iluminan la perspectiva de los estudios de la sociología de la educación, al analizar el sistema educativo como un lugar no neutral. Ambos autores consideran que la escuela tiende a reproducir el sistema social existente y que se configura como uno más de los escenarios en donde los grupos sociales se enfrentan y luchan por las posiciones de poder y de jerarquías vigentes en cada sociedad. En este punto, es importante mencionar que el sistema educativo no afecta a todas las personas de la misma forma. En este sentido, es un desafío reconocer y comprender cómo los modelos institucionales y pedagógicos se articulan con dimensiones como el género, la clase y el origen, factores necesarios a considerar en las experiencias y los efectos psicosociales de las-os sujetas-os, visibilizando el posicionamiento múltiple que constituye la vida cotidiana y las articulaciones de las relaciones de poder (Troncoso, Follegati y Stutzin, 2019).

Tomando en consideración lo anterior, se consideran algunas conceptualizaciones desde las pedagogías feministas (Belausteguigoitia y Mingo, 1999; Maceira, 2008; Martínez, 2016; Montoya, 2008; Valdés, 2013; Troncoso, Follegati, Stutzin, 2019; Seoane y Severino, 2019; Pérez, Nogueroles y Méndez, 2017; Martínez y Ramírez, 2017) con el objetivo de re-pensar los territorios educativos, buscando poner en valor aspectos relevantes que nos permitan pensar las escuelas alejadas de prácticas discriminatorias, sexistas y marginadoras. 


\section{Maneras de entender las pedagogías feministas y posibles anclajes}

Si bien la convergencia y diálogo entre referentes latinoamericanas y de otros territorios puede parecer un desafío complejo, en el escenario actual se vuelve necesaria una polifonía de voces que permita conformar un mapa situado, tanto de los procesos de aprendizaje, como de los discursos feministas. Lo anterior, permite articular una reflexión que se nutra de distintas corrientes y las ponga en diálogo crítico.

Algunos elementos relevantes que aportan a esta discusión, son los propuestos por Rita Segato quien, al referirse a los aportes del feminismo europeo dentro del contexto latinoamericano, pone de manifiesto una configuración en donde el problema de la dominación de género, de la dominación patriarcal, se considera como universal, sin mayores diferencias (Segato, 2014). Esa situación llevaría a invisibilizar problemáticas específicas de los territorios no europeos, haciendo un llamado a la consideración crítica de autoras que Segato define como blancas, para de esta forma, no perder de vista la necesidad de contextualizar los discursos feministas que posteriormente pueden ser parte de una configuración pedagógica. En este sentido, es posible tensionar las aportaciones de los feminismos europeos en un contexto chileno-latinoamericano para identificar el alcance y aplicabilidad de estas propuestas en nuestro cotidiano.

En este punto se vuelve necesario mencionar, que los planteamientos de las pedagogías feministas no tendrán un posicionamiento ni una mirada única, sin embargo, es posible identificar algunos principios que se reiteran y permanecen, los cuales se consideran como anclajes significativos dentro de los discursos que constituyen a las mismas.

Principios como la experiencia, partir de las propias historias de vida en contextos singulares de aprendizaje, hacerlo en relación, poseer un sentido colaborativo de los procesos educativos, todo bajo dinámicas de cuidado y de la consideración de la enseñanza como una experiencia amorosa que propicie espacios de confianza (Montoya, 2008). Otro aspecto importante es la atención a la construcción del conocimiento alejado de prácticas discriminatorias o marginadoras, que se suscitan en la concepción de escuela marcada por la hegemonía patriarcal que reproduce situaciones de desigualdad que alimentan estereotipos sociales alejados de la discusión de la educación para la formación en libertad y del desarrollo personal de habilidades e intereses sin imposiciones o como señalan Seoane y Severino, la pedagogía feminista como potencia "transformadora y liberadora" $(2019$, p.4)

Esto se puede relacionar con lo que Pierre Bordieu define como Habitus, que será la posibilidad de llevar a cabo "productos-pensamientos, percepciones, expresiones, acciones, que tienen siempre como límites las condiciones de su producción, histórica y socialmente situadas" (Bordieu, 1991, p.96). Como indica Griselda Hernández "las costumbres, las prácticas, el sentido común, entre otros originados por los habitus en las primeras experiencias de vida durante el proceso de socialización, adquieren un sentido de naturalidad" (2013, p.91).

Es dentro de este habitus, donde emergen aspectos relativos al género, en cuanto a intereses, comportamientos o incluso aspectos físicos que están permeados con nociones de lo masculino y lo femenino, las cuales al ser dotadas y sostenidas por relaciones y 
prácticas de poder específicas dentro de su contexto (Tipler y Ruscher, 2019), permiten continuar reproduciendo discursos de la cultura hegemónica.

En este punto, es necesario hacer algunos alcances que permitan abordar una concepción de prácticas pedagógicas feministas que consideren la diversidad de género. Es relevante poner enfásis, como ya se ha mencionado anteriormente, en como las identidades de género son construidas y enunciadas contextual y relacionalmente. Para profundizar este punto, es coherente reconocer el género desde la noción de performatividad planteada por Judith Butler (1988). Desde la perspectiva de Butler, la identidad de género se construye a través de la repetición de actos estilizados que son simultáneamente "intencionales y performativos" (Butler, 1988, p.522), situando la dimensión de género como una construcción sostenida por actos en contextos particulares. Dado que esta "perfomatividad no es un acto único, sino una repetición y un ritual que consigue su efecto a través de su naturalización en el contexto de un cuerpo" (Butler, 2001, p.17). Este concepto se relaciona con la idea de habitus (Bourdieu, 1999) donde como ya se menciono anteriormente, el orden social se va a inscribir en los cuerpos. Para este autor el habitus implica que la representación del cuerpo debe sujetarse a una etiqueta o norma impuestas desde el ámbito social, lo que va a implicar una manera de llevar y de ser cuerpo, la cual se va a perpetuar en la relación de éste, con el entorno. Es a través de estos procesos, que se van configurando "las relaciones de poder, históricas y socioculturales entre mujeres, varones, y otras identidades de género" (Ministerio de Educación, 2015, p.9).

Esto es relevante en el contexto educativo, dado que una mirada desde la perfomatividad del género permite, como explicita Marta Lamas hacer referencia a los procesos de diferenciación, dominación y subordinación entre varones y mujeres obliga a remitirse a la fuerza de lo social, y abre la posibilidad de la transformación de costumbres e ideas (Lamas, 2013), aspectos centrales de un posicionamiento docente feminista, que se vuelve transversal al momento de hablar de una práctica pedagógica situada, respetuosa y en consideración de las diversidades dentro del aula.

Pueden parecer evidentes estos aspectos tanto en la formación, como en el ejercicio profesional docente, en donde es esencial el aspecto relacional, cotidianamente presente en la praxis, sin embargo, en el contexto de las pedagogías feministas se evidencian y explicitan estos puntos como parte de estrategias metodológicas y didácticas que van encarnando la práctica pedagógica desde posturas feministas diversas y por tanto, será esa misma diversidad la que contextualice las prácticas docentes en espacios específicos. En consecuencia, se considera a las pedagogías feministas y los aspectos destacados en este artículo, más como una brújula reflexiva, una concepción, un proceder y no como un conjunto específico de pasos a seguir.

Es posible entonces comenzar una reflexión que pueda identificar los puntos de partida de estos planteamientos en los contextos educativos contemporáneos.

Uno de estos puntos fue la identificación de incomodidades históricas que abrieron un espacio a la consideración de las pedagogías desde los pensamientos feministas. Principalmente, como plantean Belausteguigoitia y Mingo (1999), el descontento de percibir cómo el sistema patriarcal permea diversos ámbitos de las sociedades, desembocando 
en una construcción social marcada por el patriarcado, y que tiende a la reproducción de los mismos. “¿Qué tipos de cartografía teórico-metodológica ubica a los vectores de la diferencia (género, sexualidad, clase y raza) en el interior de los discursos educativos con el fin de favorecer la crítica de complejos sistemas de dominación?" (Belausteguigoitia y Mingo, 1999, p.14). Esta interrogante emerge en el escenario actual, comprendiendo que la escuela "como toda institución social, refleja las visiones sociales predominantes y, por ende, puede reproducir prácticas discriminatorias" (Valdés, 2013, p.56). Esta idea toma fuerza, al advertir que es precisamente en los espacios escolares donde existe una importante presencia de prácticas discriminatorias por género y clase (Tomé y Rambla, 2001). Sumado a lo anterior, se reviste de valor lo que Connell denomina la Feminidad Subrayada para referirse a un "ideal cultural más extendido sobre la mujer que acentúa su sociabilidad, su fragilidad, su pasividad, la aceptación de los deseos del hombre y receptividad sexual" (Hernández y Reybet, 2008, p.55), una dinámica de subordinación desde el sistema masculino dominante, que refuerza la superioridad del mismo en los diversos espacios sociales.

Esto se puede relacionar también con la propia pedagogía, puesto que también se posiciona y plantea discursos que contextualizan y orientan la educación, comprendiendo que "que sustenta y promueve la ética y un objetivo político" (Maceira, 2008, p.28), por tanto, se volvería indispensable la relación entre las luchas feministas y el ejercicio pedagógico para el avance en la igualdad de derechos civiles y la toma de decisiones de los colectivos más desfavorecidos socialmente.

En este sentido, hablar de un discurso pedagógico es hablar también de un posicionamiento ético y político que, en el caso de las pedagogías feministas, se plantea desde la distancia con el patriarcado y en actitud propositiva, colaborativa e integradora, facilitando formas de comprender los procesos pedagógicos que no se limitan al quehacer de las escuelas formales.

De esta manera, las pedagogías feministas en contextos diversos podrían ser pensadas con énfasis en lo que Jennifer Gore (1996) plantea como la atención en las formas en que se produce el conocimiento y las propias experiencias del aprendizaje marcadas por elementos relacionados al género.

Jennifer Gore plantea en su libro "Controversias entre las pedagogías" su pensamiento sobre la palabra 'pedagogía', y señala: "Aunque muchos relacionan exclusivamente la palabra 'pedagogía' con el campo de la educación formal, en realidad, se trata de una actividad referida a todos los aprendizajes, a toda la producción de saber" (Gore, 1996, p.14). Este planteamiento sobre la pedagogía lo consideró como una reflexión a modo de conclusión luego de que en 1992 comenzara a preguntarse ¿Qué son las pedagogías feministas? o ¿Qué hace que las pedagogías sean efectivamente feministas? en su monográfico "La ética foucaultiana y la pedagogía feminista".

Según Gore las definiciones dependerán del punto de vista. Por un lado, los contextos sobre estudios de la mujer se inclinan por preguntarse “Qué es la pedagogía feminista?" (Gore, 1992, p.158). Mientras que desde los contextos educativos la pregunta se plantea desde: “Qué hace que la pedagogía sea feminista?” (Gore, 1992, p.158). Esto permite 
reflexionar, sobre que independiente de cómo se planteen las preguntas, las respuestas no serán únicas y tendrán múltiples factores que llevarán a las personas no sólo a pensar en la diversidad de pedagogías feministas, sino que a pensar los aprendizajes de maneras contextualizadas, híbridas y mutables.

En relación a las preguntas planteadas anteriormente y según los grupos de estudios de la mujer planteados por Gore, los cuales se inclinan por hacerse preguntarse sobre qué es la pedagogía feminista, ellas centran la mirada en "Cómo estudiar y qué estudiar" (Gore, 1992, p.161), haciendo énfasis en la propia experiencia, la que no se limita a la escuela: "No puedo hacer una distinción tan clara, aquí está la educación y aquí la vida, porque es un proceso continuo" (Rodríguez, 2002, p.53). De las evidencias y los planteamientos de los estudios de la mujer derivan más del "movimiento de liberación de la mujer y de las actividades de concienciación que del estudio académico formal de la Educación" (Gore, 2002, p.161). Sin embargo, ese distanciamiento de la educación formal, hace que esta perspectiva se aleje del espacio educativo formal.

Por otro lado, los grupos de profesionales de la educación que trabajan sobre las pedagogías feministas se centraron en la práctica educativa y la reflexión en torno a ella, pensamientos que nacen como respuesta a la tradición patriarcal que regía las escuelas y espacios de aprendizaje.

Esta corriente de pedagogía feminista rechaza ampliamente el sentido más técnico o de instrucción de la pedagogía, la pedagogía de cómo enseñar, y en su lugar aborda la pedagogía desde un enfoque más amplio, insistiendo en cómo se produce el conocimiento y la experiencia de género. (Gore, 2002, p.162)

Centran sus reflexiones en la práctica pedagógica y en cuestiones de género. “También en la mediación entre las propias inquietudes, curiosidades, las personas y los conocimientos" (Montoya, 2008, p.42). Lo anterior, permite ampliar la práctica pedagógica a la cotidianeidad del fuera de la escuela.

Según las evidencias entonces, en las pedagogías feministas, existen puntos en común entre las diferentes corrientes de pensamiento, ya sea desde los estudios de la mujer o desde la corriente pedagógica y es que se reconoce que las pedagogías feministas están necesariamente contextualizadas a momentos y lugares determinados, por tanto es muy difícil comparar unos con otros.

Cada lugar determina la práctica de sus pedagogías y las maneras en que las personas llevan a cabo los procesos de aprendizaje, de forma dinámica y situada, lo que se alinea con el pensamiento de las feministas postestructuralistas que visualizan las pedagogías feministas como métodos que escapan a esencialismos "prófugas no sólo de campos que las ignoran, sino también de significaciones (incluso feministas) que las empobrecen al fijarlas a fórmulas estrictas, tanto del señalamiento de las particularidades de la opresión como de los mecanismos y formas de liberación" (Belausteguigoitia y Mingo, 1999, p.19). Esto también implica cuestionar constantemente el medio educativo formal y sus posibles reproducciones de dominación. Es así como se visualiza una ten- 
sión entre "la educación como liberadora y la educación justamente como lo contrario, como la reproductora de la opresión y de todo aquello que lo libera" (Belausteguigoitia y Mingo, 1999, p.18).

Según lo planteado, pensar el sistema educativo como un espacio de dominación, podría ser una oportunidad de cambio desde las propias bases que conforman las instituciones educativas.

Aún cuando las investigaciones que se han ido nombrando en este artículo dan cuenta de las potencialidades del trabajo en centros educativos, los esfuerzos en torno a las pedagogías feministas en las escuelas son aún insuficientes, aún cuando las movilizaciones feministas de mayo 2018 (Reyes-Housholder y Roque, 2019; Richard, 2018; Troncoso, Follegati, y Stutzin, 2019; Undurraga y Simburgüer, 2018), marcaron un punto de inflexión en las principales discusiones referidas a educación no sexista, la discriminación por género y la organización de escuelas diferenciadas de varones y mujeres.

En relación a la consideración de lo femenino en el proceso de aprendizaje, Adrienne Rich (1978) hace un llamado a responsabilizarnos del ser mujer y las características que conlleva: "Creyendo en el valor y la significación de la experiencia, las tradiciones y las percepciones de las mujeres. Pensando seriamente en nosotras mismas, no como en un muchacho, no como neutras o andrógenas, sino como mujeres" (Rich en Gore, 1992, p.168). Por otra parte, reflexiona en torno al compromiso de las propias mujeres de formar mujeres: "Lo más importante que una mujer puede hacer por otra es iluminar y ampliar el ámbito de sus posibilidades” (Rich, 1978, p.243).

\subsection{Cruces con la pedagogía crítica}

Una vinculación interesante es la que se puede llevar a cabo entre las pedagogías crítica y feministas, puesto que ambas cuestionan los sistemas de opresión, lo que nos podría llevar a pensar en puntos comunes, como la invisibilización de la diferencia en el contexto escolar, dinámicas autoritarias, clasistas y discriminatorias en general, orientadas a la competencia y no a la colaboración y aprendizaje colectivo.

La pedagogía crítica cuestiona las relaciones de poder y dominación que existen dentro de la escuela. Plantea que el proceso educativo debe estar constantemente reflexionando de manera crítica.

La pedagogía crítica ve la educación como una práctica política social y cultural, a la vez que se plantea como objetivos centrales el cuestionamiento de las formas de subordinación que crean inequidades, el rechazo a las relaciones del salón de clases que descartan la diferencia y el rechazo a la subordinación del propósito de la escolarización a consideraciones económicas. (González, 2006, p.83)

En el contexto de América Latina, este proceso ha ido acompañado de acontecimientos socioculturales, sometimiento y violación de derechos humanos fundamentales, que han hecho crecer con fuerza el pensamiento de la pedagogía crítica como medio para el cambio social. 
Enseñar con fines de transformación social significa enseñar a los alumnos a asumir riesgos y a luchar en las continuas relaciones de poder para ser capaces de imaginar y fomentar aquellas posibilidades todavía no realizadas con respecto a la sociedad en general que auguran un futuro más democrático y más humano. (Giroux, 1992, p.75)

La pedagogía crítica es también un espacio de participación y una manera de hacer consciente a las diversas comunidades de las situaciones de abuso y dominación, situando este trabajo reflexivo desde el aula.

Paulo Freire (1993), desarrolla en Brasil la alfabetización de masas en los campos de caña de azúcar y desde allí critica las formas de hacer educación, las maneras autoritarias y rígidas del aprendizaje y la ausencia de espacios reflexivos en relación a la sociedad, la economía y el libre mercado.

Las reflexiones sobre la educación bancaria, su autoritarismo, la educación cuestionadora, el diálogo, las iniciativas democráticas; la necesidad de desafiar la curiosidad de los educandos en una práctica educativa progresista; la presencia crítica de educadoras y educadores y de las-os educandas-os, mientras que enseñando unas y aprendiendo otros, "todos aprenden y enseñan, sin que esto signifique que sean todos iguales o que quien enseña no aprende o quien aprende no enseña" (Freire, 1993, p.171-172).

La democracia, el pensamiento reflexivo y la crítica a los sistemas sociales, llevarían según Freire (1993) a varones y mujeres a la libertad con total consciencia de quienes eran y dónde estaban. El pensamiento de Freire "sigue los fundamentos de la escuela activa, el diálogo, la crítica y la búsqueda permanente de creación de una conciencia sobre la realidad" (Freire citado en Ocampo, 2008, p.70).

Por su parte Marisa Belausteguigoitia y Araceli Mingo (1999) también dan luces sobre la pedagogía crítica y su importancia para el desarrollo educativo, sobre todo en contextos de vulnerabilidad social.

El campo de la pedagogía crítica centró sus energías en el salón de clases como espacio privilegiado para potenciar la voz de las y los estudiantes, construidos como los otros, los subalternos del proceso de conocimiento. Centró su atención en el desplazamiento del aula como espacio secuestrado por las técnicas pedagógicas aisladas del contexto político y cultural. (Belausteguigoitia y Mingo, 1999, p.28).

Con estos antecedentes las pedagogías feministas posestructuralistas reflexionan en torno a la pedagogía crítica, sus cruces y distancias, planteando que "la pedagogía crítica y el feminismo posestructuralista comparten la necesidad de analizar al otro en su intrínseca e inexorable sujetación al discurso como sistema de poder" (Belausteguigoitia y Mingo, 1999, p.31).

Giroux por su parte hace énfasis en el reconocimiento de formarnos respetando las diferencias y singularidades del alumnado y se refiere a la pedagogía de la diferencia:

Una pedagogía de la diferencia precisa abordar la importante cuestión de cómo las representaciones y prácticas de la diferencia se aprenden, interiorizan, desafían o trans- 
forman activamente. Ésta es la única forma en que los profesores pueden desarrollar una pedagogía en favor de la diferencia. (Giroux, 1992, p.79).

Lo que además podría caracterizarse por lo que Teresa de Lauretis (1987) plantea como un esfuerzo continuo para propiciar nuevos lugares de discurso, poder rescribir narrativas y dar cabida a posiciones de sujetas-os que históricamente han estado subalternizadas.

Centrando la mirada en las pedagogías de las feministas posestructuralistas a las que se hace alusión en el libro "Géneros prófugos", se puede decir que también plantean un conocimiento contextualizado, con conocimiento del entorno en el cual se desarrollan los aprendizajes, se da importancia a "la ubicación del sujeto femenino (posicionamiento) dentro de una cartografía hipercrítica de los límites, las fronteras, las definiciones y las exclusiones, llevado a cabo por los discursos fundadores (metanarrativas)" (Belausteguigoitia y Mingo, 1999, p.33).

De esta manera es posible esbozar como las pedagogías feministas se fugan de la escuela realizando un ejercicio de expansión a otros territorios y lugares, mientras que la pedagogía crítica va a centrar sus esfuerzos en la institución escolar. Sumado a lo anterior, las pedagogías críticas y feministas convergen en un permanente cuestionamiento a las esferas de poder y los dispositivos de control, con el objetivo de abrir espacio a múltiples voces, respetando y valorando la diferencia de quienes participan en los procesos de aprendizaje tanto fuera como dentro de la escuela.

Una pedagogía a favor de la diferencia no sólo busca comprender cómo se construye la diferencia en la intersección del currículum oficial de la escuela y de las distintas voces de los alumnos pertenecientes a grupos subordinados; también pone en juego todas las contradicciones que se dan en las múltiples posiciones de sujeta-o que caracterizan las subjetividades de las-os estudiantes. "Las voces que caracterizan los diversos grupos de estudiantes no forman una sola unidad susceptible de ser reducida únicamente a las categorías de clase, raza o género" (Giroux, 1992, p.80).

Jennifer Gore reafirma las ideas de Giroux señalando que "tanto los discursos pedagógicos críticos como los feministas hacen hincapié en la experiencia y la voz de los alumnos (...) están vinculados con movimientos políticos y sociales que tratan de erradicar las múltiples formas de opresión" (Gore, 1996, p.25). Por su parte el construccionismo social también cuestiona las figuras hegemónicas; "el construccionismo ofrece una base fundamental para desafiar las realidades dominantes y las formas de vida de ellas asociadas" (Gergen, 1996, p.82). Dos formas centrales para llevar a cabo este desafío son criticar la cultura y llevar a cabo una crítica interna.

Las pedagogías no se limitan a las escuelas, sino que pueden ser llevadas a cabo en cualquier contexto en donde se puedan construir espacios de pensamiento, que para la pedagogía crítica se concentran en los establecimientos educativos y desde ahí a los contextos sociales. A diferencia de las feministas posestructuralistas que plantean la idea de que este espacio de pensamiento reflexivo puede ser llevado a cabo en contextos cotidianos, valorando por tanto todas las experiencias de vida para aprender y no sólo las que 
se desarrollan en el contexto de la escuela. Sumado a pensar la pedagogía crítica como formación de masas versus, las pedagogías feministas que más allá de la cantidad de personas a las que busca formar, nace desde la diferencia sexual de una minoría social y, por lo tanto, aunque forme a una gran cantidad de personas, centra sus esfuerzos en llevar a cabo una educación consciente de la singularidad. Lo que no significa que estas tradiciones pedagógicas no puedan ser complementarias.

\section{Sobre las experiencias y los contextos}

Dentro de los planteamientos de las pedagogías feministas que se han ido revisando a lo largo del artículo, existen dos aspectos centrales, estos son las experiencias personales y los contextos cotidianos y particulares del aprendizaje.

Para comenzar a hablar de las primeras, Irene Martínez (2016) plantea que la educación asociada al feminismo debe considerar las experiencias y los saberes de las mujeres. Martínez a partir de la lectura de la "Decentralización de la teoría hegemónica de género: las implicaciones para la investigación educativa" de Fennell y Arnot (2009) y de "Bajo los ojos de Occidente: academia feminista y discursos coloniales" de Mohanty (2008), propone la consideración de las experiencias para:

1) Deconstruir los conceptos dominantes de la teoría universal del género (lenguaje, ideas, teorías, modelos...); 2) Hacer crítica sobre las simplificaciones y generalización de las narrativas dominantes acerca del significado de ser mujer en los territorios del "Sur"; 3 ) Descolonizar la diversidad de experiencias de las mujeres, sus luchas, negociaciones, resistencias, formas de opresión...; 4) Incluir puntos de vista familiares desde el entendimiento multidimensional de los roles de género; y 5) Enfatizar el poder de las mujeres desde sus culturas y experiencias propias. (Martínez, 2016, p.143)

Estos elementos, permiten reflexionar en torno a la consideración de las experiencias y los saberes desde posturas feministas como un ejercicio constante de reflexión crítica donde se implica el "aprender en relación y poseer un sentido colaborativo de los procesos de aprendizaje" (Montenegro, 2018, p.292).

A las propuestas anteriores, podemos sumar un concepto que es necesario considerar y es el partir de sí, como un partir de las propias experiencias para aprender. En palabras de Ana María Piussi (2000) este partir de sí hace referencia a intercambios de palabra y acción, a transformar esa experiencia personal en un proceso constante de mediación.

Poniendo en juego deseos pasiones, experiencias personales, más allá de las mediaciones ya dadas, casi siempre lejanas, impersonales y fuera de contexto, que se superponen a la realidad de las cosas y la tornan rígida, impidiendo, en suma, que se sea una buena mediación (Piussi, 2000, p.113). 
Una manera de aprender que se presenta coherente para construir conocimiento y hacer que sea un acto de mediación con el aprendizaje de otras personas, "así la noción de experiencia aparece como testimonio subjetivo, como la auténtica clase de verdad" (Arfuch, 2010, p.72) y comienza una relación entre narrativas y lectoras-es donde la propia historia de vida se intersecciona con la lectura de otras personas, de manera que "el receptor[a] cuando sigue una historia amplían el horizonte de su existencia y modifican su pautas de comportamiento, así como su precomprensión temporal" (Foucault citado en Ricoeur, 1999, p.216) estableciendo relaciones también con las experiencias de otras-os, elaborando un tejido de saberes colectivos que tiene la posibilidad de dar paso a un reconocimiento de las trayectorias personales en contextos de aprendizaje.

Socializar y exteriorizar las vivencias de manera oral o escrita, permite no sólo construir un conocimiento propio, sino también aportar a la construcción del aprendizaje de otras-os. Una razón más para compartirlas a través de relatos, y de esa manera "la historia de la vida se convierte, de ese modo, en una historia contada" (Ricoeur, 1999, p.216), otro aspecto relevante que configura las pedagogías feministas: los relatos, narrativas autobiográficas y pequeñas narrativas. Esto también dependerá de cuáles hayan sido nuestras trayectorias vitales que serán contadas, comprendiendo que "lo que vemos depende del lugar en el cual nos situamos al mirar" (Bárcena, 2004, p.174), aspecto que da singularidad a nuestros relatos y da importancia, coherencia y sentido a los contextos diversos y las miradas desde donde nos posicionamos.

Las experiencias serán entonces, actos de reconocerse en relación con otras personas, asumiendo por tanto, que la propia experiencia es importante para las relaciones educativas y para la construcción del conocimiento. Legítima y verdadera en el sentido de relación honesta con otras personas y dando el mismo sentido a nuestras experiencias.

Me descubro partiendo de mí cuando digo la verdad y sé que lo es porque mi cuerpo no se tensa y mi voz sale más fluida. La verdad que he llevado al aula tiene que ver con mantener y cuidar la relación para que sea verdad lo que hacemos porque vivir de verdad en el aula significa que lo que allí acontezca tenga sentido para mí, y para cada cual, si se deja. (Arnaus, 2007, p.125-126)

Entender que los posicionamientos subjetivos no restan seriedad a los procesos formativos. Hablar desde la subjetividad da consistencia a los discursos, dejando en evidencia que han sido vividos por personas que ahora, buscan propiciar el diálogo. En definitiva, permite reconocer a las personas y sus singularidades, comprendiendo a su vez que las-os individuas-os son multidimensionales y con experiencias particulares, tal cual refiere González (2009) "la recuperación del sujeto que piensa implica la integración de las emociones, la fantasía y la imaginación en el desarrollo intelectual. No hay desarrollo intelectual separado del desarrollo de la persona" (p.4). Aquí se vuelve pertinente la consideración de los pequeños relatos autobiográficos como nudo entre la vida pública y privada en la que se transita en los espacios de aprendizaje, como señala Arfuch (2010) el espacio Público/biográfico: 
La articulación indisociable entre el yo y el nosotros, los modos en que las diversas narrativas pueden abrir, más allá del caso singular y la "pequeña historia", caminos de autocreación, imágenes e identificaciones múltiples, desagregadas de los colectivos tradicionales, y afianzar así el juego de las diferencias como una acentuación cualitativa de la democracia. (Arfuch, 2009, p.80)

De esta manera, los relatos serían una constante relación con la experiencia, que en palabras de Jorge Larrosa es "la posibilidad de que algo nos pase, o nos acontezca, o nos llegue" (2003, p.174).

Con estas reflexiones los procesos de aprendizaje propuestos por las pedagogías feministas serían también una invitación a dejarse llevar y estar con la disposición a experimentar con los sentidos y desde aquí hacer que esas experiencias adquieran sentido a través de la reflexión que también se plantea como un proceso dinámico que va cambiando, mutando y regenerándose a lo largo de la vida. Como señalan Contreras y Pérez de Lara "hacer significativo lo vivido es más un movimiento que un logro, más una necesidad que una conclusión, más una búsqueda que una certeza" (Contreras y Pérez de Lara, 2010, p.36). Por su parte Bozhovich (1981) reconoce también el valor de las propias experiencias para formarse y aprender "para comprender exactamente que influencia ejerce el medio sobre el niño y, por consiguiente, como determina el curso de su desarrollo, hay que comprender el carácter de las vivencias del niño, el carácter de su relación afectiva con el medio" (Bozhovich, 1981, p.123).

Con esto también es necesario comprender que los puntos de partida y las experiencias son también dinámicas en el tiempo, siendo entonces "una unidad fluida de vida y pensamiento" (Molina, 2011, p.118) que está en constante reflexión.

El sentir -como el pensar poniendo en juego la propia experiencia de vivir- no es algo abstracto o cognitivo sin sede alguna; el sentir está enraizado con el cuerpo, que es su sede. Y digo cuerpo como entramado de lo orgánico, lo físico, lo psíquico, lo energético que se combina con gracia y azar de manera singular y sexuada: Hombre o mujer. (Arnaus, 2007, p.55)

Para Contreras y Pérez de Lara (2010) es importante también la idea de 'experiencia educativa', planteando que investigarla significa "adentrarse en los mundos subjetivos, inciertos, ligados al acontecer, a lo singular, y desde ahí, tratar de sostener, en primera persona, la pregunta sobre su sentido educativo" (p.45), esto tanto dentro, como fuera de la escuela. Esta idea es reafirmada por Van Manen al señalar que "las historias personales inducen a la reflexión pedagógica" (Van Manen, 1998, p.63) y es precisamente por esto que son tan importantes en los procesos pedagógicos feministas.

Son importantes también todas las reflexiones desplazadas que nacen a partir de las experiencias pues, si no tenemos espacios de reflexión y pensamiento sobre ellas, no podremos construir conocimiento. Experiencias que a su vez se deben relacionar con conocimientos más objetivos como los planteados por la escuela. La experiencia y el conocimiento académico carecen de sentido si no somos capaces de relacionarlas. Por otro lado, es importante destacar que la experiencia es mucho más basta que el pensamiento, tal cual señalan Contreras y Pérez de Lara (2010): 
La experiencia es irreductible a pensamientos y siempre supone una multidimensionalidad y simultaneidad de las que es imposible dar cuenta ajustada en el pensar. El pensamiento ordena, elige, muestra aspectos que destaca, a los que atribuye significado, pero no puede decirlo todo. (p.37)

Según lo planteado por estos autores el significado de las vivencias está dado en la medida en la cual se transforma en experiencia, al verbalizarla, compartirla y al darle sentido junto a otros.

De esta manera y en concordancia con lo planteado anteriormente, la experiencia ayuda a construir conocimiento en la medida en que la contextualizamos y relacionamos con otros conocimientos, propios o dados por otras personas.

La experiencia involucra entonces, todos nuestros sentidos, nos ayuda a relacionarnos con otras personas para llevar a cabo los procesos de aprendizaje, siendo la escucha una de las formas de comunicación más importantes tanto de proceso de aprendizaje como de los posicionamientos feministas que apelan a la escucha como acto de consideración y atención a la-el otra-o.

La escucha "está desorientada, es decir, privada de referencias, abierta a cualquier imprevisto. Es una posición interior. Esta disposición a la escucha del otro permite captar la diferencia de la que son portadores la experiencia y el tiempo de los niños" (Puleo, 2009, p.114). Dialogar es construir también experiencias, indispensables en el proceso pedagógico, sin embargo, no siempre se le da la importancia que debe tener. Elizabeth Ellsworth lo explica:

Lo que se escapa a la mayoría de discusiones sobre el diálogo en educación es esto: el diálogo -como práctica de enseñanza defendida a lo largo de la bibliografía educativa- es en sí mismo una relación socialmente construida y políticamente interesada. (2005, p.58).

Aspecto relevante dentro de las pedagogías feministas como una forma de dar valor a los espacios dialógicos y comunitarios para aprender. Ellsworth (2005) plantea también que el diálogo como pedagogía carece de neutralidad, por tanto, los procesos de aprendizaje son subjetivos, entendiendo que quienes se involucran en el proceso vienen con una carga experiencial que les permite opinar y reflexionar desde ahí. Es habitual que se busque esa neutralidad que Ellsworth plantea como inexistente en la pedagogía para buscar el traspaso de conocimientos.

\section{Formarse en femenino y en relación}

Otro aspecto importante que emerge como fundamental para las pedagogías feministas, tienen relación con la formación en relación desde vínculos colaborativos, que dejen en evidencia las colaboraciones, los vínculos y la admiración que podemos experimentar al momento de trabajar con otras personas. Esto planteado desde relaciones de confianza que permitan una relación legítima con quienes estoy llevando a cabo procesos 
de aprendizaje. Aquí volvemos a la noción de experiencia que para Braidotti es "uno de los conceptos clave de las teorías feministas, pero referido a la experiencia de las mujeres en la vida real" (Braidotti citada en Acaso, Ellsworth y Padró, 2011, p.34), mientras que para Luke (1999) existe una experiencia que necesariamente se vincula con la vida cotidiana, que Consuelo Flecha (2006) vincula con una forma de ponerse en juego "para crear y vivir en lo cotidiano relaciones de libertad, como el saberte acompañada por las experiencias de otras mujeres que han ido tejiendo en tu biografía esa trama que te habla de un origen propio" (p.46).

Según esto, el proceso de aprender en femenino se podría pensar como un proceso entre mujeres, sobre mujeres o para mujeres. Sin embargo, la propuesta de las pedagogías feministas es a la consideración de las personas desde prácticas feministas del cuidado, la confianza y la construcción del aprendizaje en comunidad, valorando la singularidad del otra-o y por tanto no exclusiva de mujeres.

Estos principios podrían ser aplicables a cualquier entorno educativo, tanto dentro como fuera de la escuela, tanto con varones, mujeres o personas con identidades y géneros diversos. Por tanto, podemos aprender en femenino sin ser mujeres o educarnos entre mujeres o escuelas exclusivas de mujeres sin aprender en femenino. Esto último es bastante común en Chile donde las escuelas que forman mujeres pasan por alto la singularidad y las preocupaciones propias y siguen formando en contexto neoliberal, androcéntrico y patriarcal, características que fueron cuestionadas con los movimientos sociales de 2018 conocidos como 'Mayo Feminista'.

¿Qué hicieron las tomas feministas de mayo 2018? Reemplazar el ideologismo neoliberal de la "calidad" como un término vaciado de toda referencialidad social y cultural (un término abstracto-neutral cuya indefinición de contenidos garantiza su aplicabilidad general según los indicadores de gestión que promueve el "capitalismo académico" de la universidad globalizada) por la demanda libertaria de una "educación antisexista". (Richard, 2018, 117)

Esto nos hace pensar en lo que la propia Nelly Richard (2018) definiría como un slogan -a educación antisexista- de las movilizaciones feministas que pondría sobre la mesa cuestionamientos relativos a la crítica teórica, políticas y cultura que pondría en entredicho las estructuras de poder y representación de lo masculino como lo dominante o la norma para pasar a una gama infinita de proposiciones sociales de cómo vivir en ella, alejándonos de la desigualdad por género e identidades.

Volviendo a la idea de los aprendizajes en relación y según la corriente del feminismo italiano ${ }^{1}$ la primera relación que tendríamos que reconocer es con la madre.

\footnotetext{
${ }^{1}$ La librería de mujeres de Milán, Italia es la responsable de dar a luz numerosas investigaciones que se vinculan con el orden simbólico de las madres, aprendizajes en relación entre mujeres, relaciones de confianza y autoridad femenina entre otros temas.
} 
Casi nadie nota que cada criatura que nace es humanizada en una relación, así cuando su madre, libremente, le enseña a hablar y, al enseñarle a hablar, le enseña el mundo y le transmite la importancia del vínculo para vivir y convivir. (Rivera, 2012, p.24)

Teniendo entonces esta primera relación entre madres e hijas e hijos y la idea de mostrarles el mundo desde la confianza, es que se hace necesario también las relaciones con tratos amorosos (Montoya, 2008), relaciones que se basan en la libertad, la seguridad y el respeto como actos civilizadores.

Relaciones de este tipo propiciarían los aprendizajes de maneras orgánicas, alejadas de dinámicas del miedo y considerando la multidimensionalidad de quienes participan de estos procesos.

Hablar de aprender en femenino es también hablar de establecer lazos afectivos entre mujeres, lo que lleva a establecer relaciones de confianza y a estar mucho más llana a construir el conocimiento, recibir críticas y compartir lo que se sabe. Desde aquí es mucho más fácil hacer amistad a la manera de las mujeres. Hace sentido hablar de la palabra affidamento, término que se refiere a juntarse entre mujeres para compartir, aprender, admirar y llevar adelante diversas luchas. Según el texto 'No creas tener derechos' de la Librería de mujeres de Milán (1991) affidarse sería una relación social como contenido e instrumento esencial para las luchas feministas, comprendiendo que la subjetividad femenina en palabras de Porter (1999) le da un importante valor a las relaciones de amistad, lo que facilitaría los procesos de aprendizaje de habilidades vinculadas con aspectos sensibles del ser, entendiendo que "la idea feminista de la amistad exige, en primer lugar, la consideración de la confianza en una misma; en segundo, que esto contribuya al crecimiento moral, y, por último, que la síntesis de la atención personal y la autonomía confirme la amistad activa" (Porter, 1999, p.83).

En este sentido entonces, las relaciones de amistad que se van enriqueciendo a lo largo del tiempo y la cotidianeidad apuntan al desarrollo compartido de habilidades y aprendizajes, propiciando también el cuidado y preocupación entre quienes mantienen cercanía. Existe, por lo tanto, un compromiso entre las personas para avanzar en los aprendizajes de la escuela y ofrecer apoyo cuando a una de las amigas o amigos le cuesta más un contenido que otro.

Lo que diferencia las amistades de las mujeres del sentido desconectado del yo y el otro de los hombres son las intimidades compartidas, el apoyo mutuo que surge de la vida cotidiana concreta y una parcialidad preocupada y una responsabilidad con respecto a las otras personas, relaciones y contextos concretos. (Luke, 1999, p.28)

Aprender en femenino y en relación es basar los aprendizajes en la confianza que las mujeres podemos construir entre nosotras. Dar espacio a la creatividad y la generosidad de pensar que otras personas también pueden enseñar-se, es entonces un acto de humildad y reconocimiento de autoridad femenina entre pares con las que se van desarrollando relaciones cotidianas en femenino que permiten visibilizar nuevas maneras de construir el conocimiento y hacer escuela. 


\section{Para finalizar}

Comprendiendo el carácter dinámico de las propuestas presentadas en este trabajo, este ensayo se configura pricipalmente como una invitación a poner en el centro de la reflexión las posibilidades que nos brindan las Pedagogías Feministas en torno a la tranformación tanto de la escuela, como del sistema educativo, "como un conjunto de procesos y de relaciones de autoridad (que no autoritarismo), donde se reconoe y se respeta al otro y a la otra" (Martínez, 2016, p.141). Fisurar procesos de aprendizaje que se encuentran históricamente invisibilizados y subordinados a un contexto cargado de hegemonía masculina; es justamente una apelación a comprender la importancia de observar el ejercicio docente, la formación inicial y los procesos de aprendizaje desde la consideración de las singularidades, las propias trayectorias vitales y las demandas sociales para una formación consciente de las responsabilidades y posibilidades de cambio, el valor por las personas y la comprensión de la escuela y los espacios de formación como lugares de transformación, dinámicos, y en constante movimiento donde el valor de la comunidad se nos presenta, junto al poder de la construcción de conocimiento, el cual es necesario que se produzca en direcciones diversas, ausente de jerarquías para un aprendizaje situado y significativo.

Sin duda las Pedagogías Feministas estimulan las relaciones de solidaridad y colaboración entre mujeres y sujetos no hegemónicos. De esta manera, se abren caminos orientados al fortalecimiento de los procesos colectivos y conjuntos, que a la vez potencian el desarrollo de procesos y potencialidades singulares. Aunque no hay duda alguna, de que son procesos que estarán atravesados de forma permanente por tensiones, avances y retrocesos, y que implica un ejercicio constante y permanente de reflexión sobre la práctica docente y pedagógica en general, propiciando un "re-pensamiento constante de los medios educativos formales" (Montenegro, 2019, p.115) además de las relaciones entre los procesos de aprendizaje y los contextos cotidianos

Por ello queda insistir en la potencia e importacia de relevar las Pedagogías Feministas como una clave necesaria para reorganizar el ejercicio pedagógico, en donde se pueda dar valor a la relación, a las experiencias cotidianas y locales, al aprendizaje no centrado solamente en la cognición, a la colaboración y la comunidad que finalmente es donde se pone en juego de forma cotidiana la construcción de lo social. Sin duda queda un auspicioso y desafiante camino por recorrer.

\section{Referencias}

ACASO, MARÍA, ELLSWORTH, ELIZABETH Y PADRÓ, CARLA. (2011). El aprendizaje de lo inesperado. Madrid: Catarata.

ARFUCH, LEONOR. (2010). El espacio biográfico. Dilemas de la subjetividad contemporánea. Buenos Aires: Fondo de cultura económica. 
ARNAUS, REMEI. (2007). Partir de sí. Hacer escuela en la nueva civilización. En Montoya, María Milagros. (Ed.), Saber es un placer. La práctica política de mujeres que buscan dar sentido libre a la educación (pp.123-140). Madrid: Horas y horas.

AZUAZ, XIMENA. (2016) Aprender a ser mujer, aprender a ser hombre. La escuela como reproductora de estereotipos (37-46). En Educación No Sexista. Hacia una Real Transformación. Santiago: Red Chilena Contra la Violencia hacia las mujeres.

BÁRCENA, FERNANDO. (2004). La práctica reflexiva en educación. Introducción a la filosofía de la educación. Madrid: Universidad Complutense de Madrid.

BELAUSTEGUIGOITIA, MARISA Y MINGO ARACELI. (1999). Fuga a dos voces. Ritmos, contrapuntos y superposiciones del campo de los estudios de género y la educación. En Belausteguigoitia, Marisa y Mingo, Araceli (Comp.), Géneros Prófugos (pp.13-53). México: UNAM.

BERNSTEIN, BASIL. (1994). La estructura del discurso pedagógico Madrid: Morata.

BERTARELLI, PAULA. (2014). Cuerpos que irrumpen en la escuela: actos de género y procesos de diferenciación entre jóvenes mujeres. En Paulín, Horacio y Tomasini, Marina (Ed.) Jóvenes y Escuela, relatos sobre una relación compleja (p.171-198). Argentina: Editorial Brujas.

CABELLO, CRISTEVA. (2018). Educación no sexista y binarismo de género. Agitaciones feministas y disidencias sexuales secundarias en la escuela. En Zerán, Faride (Ed.) Mayo Feminista. La rebelión contra el patriarcado (p.21-34). Santiago: LOM.

BORDIEU, PIERRE. (2000). La dominación masculina. España: Editorial Popular.

- (1999). Meditaciones pascalianas. Barcelona: Anagrama.

- El sentido práctico. Madrid, Tesaurus.

BORDIEU, PIERRE Y PASSERON, JEAN-CLAUDE. (2009). Los herederos. Los estudiantes y la cultura. Buenos Aires: Siglo XXI.

BOZHOVICH, LIDIA. (1981). La personalidad y su formación en la edad infantil. La Habana: Pueblo y educación.

BUNN, MATTHEW, THREADGOLD, STEVEN Y BURKE, PENNY JANE. (2019), Class in Australian higher education: The university as a site of social reproduction. Journal of Sociology.

BUTLER, JUDITH. (2001). El género en disputa. El feminismo y la subversión de la identidad. México: Paidós.

- (1988). Performative acts and gender constitution: An essay in phenomenology and feminist theory. Theatre journal, 40(4), 519-531.

CABELLO, CRISTEVA. (2018). Educación no sexista y binarismo de género. Agitaciones feministas y disidencias sexuales secundarias en la escuela p.21-34). En Faride Zerán. (Ed.), Mayo Feminista. La rebelión contra el patriarcado. Santiago: LOM.

VVAA. (2018) Educación no sexista. Revolución feminista. Santiago: Editorial Aún creemos en los sueños.

CONNELL, RAEWYN. (1987). Gender and power. Standford: Standford. University Press.

CONTRERAS, JOSÉ Y PÉREZ DE LARA, NURIA. (2010). Investigar la experiencia educativa. Madrid: Morata. 
DE LAURETIS, TERESA. (1987). Technologies of Gender. Bloomington: Indiana University Press.

ELLSWORTH, ELIZABETH. (2005). Posiciones en la enseñanza: diferencia, pedagogía y el poder de la direccionalidad. Madrid: Akal.

FLECHA, CONSUELO. (2006). Genealogía. En Piussi, Ana y Mañeru, Ana (Ed.) Educación, nombre común femenino (pp.46-65). Barcelona: Octaedro.

FREIRE, PAULO. (1993). Pedagogía de la esperanza. Un reencuentro con la pedagogía del oprimido. México D.F.: Siglo XXI.

GERGEN, KENNETH. (1996). Realidades y relaciones: aproximaciones a la construcción social. Barcelona: Paidós.

GIROUX, HENRI. (1992). Más allá de la teoría de la reproducción. Hacia una pedagogía de la política de la diferencia. En Giroux, Henri y Flecha, Raúl. (Ed.), Igualdad educativa y diferencia cultural (pp.53-90). Barcelona: El Roure.

GONZÁLEZ, FERNANDO. (2009). La significación de Vygotski para la consideración de lo afectivo en la educación: las bases para la cuestión de la subjetividad. Revista Actualidades investigativas en educación, vol. 9, 1-24.

GONZÁLEZ, LUIS. (2006). La pedagogía crítica de Henri A. Giroux, Revista electrónica Sinéctica (29), 83-87.

GONZÁLEZ, VICTORIA. (2010). Interpretando el pensamiento complejo: un acercamiento a Lev S. Vygotsky. Revista Posgrado y sociedad, 10(1), 38-63.

GORE, JENNIFER. (1996).Controversias entre las pedagogías. Madrid: Morata. - (1992). La ética foucaultiana y la pedagogía feminista. Revista de Educación, 155178.

GRAIZER, ÓSCAR Y NAVAS, ALMUDENA (2011). El uso de la teoría de Basil

Bernstein como metodología de investigación en Didáctica y Organización Escolar. Revista de educación, 356, 133-158.

HERNÁNDEZ, GRISELDA. (2013). Habitus, estereotipos y roles de género. Percepciones de profesores y estudiantes, Revista Docencia Universitaria, vol. 14, 89-105.

HERNÁNDEZ, ADRIANA Y REYBET, CARMEN. (2008). Ruidos y murmullos: las configuraciones discursivas que regulan las prácticas escolares. En Morgade, Graciela y Alonso, Graciela. (Comp.), Cuerpos y sexualidades en la escuela (pp.43-64). Buenos Aires: Paidós.

JOSE, JUSTIN y SHANUGA, CHERAYI. (2018). Inclusive Properties of School Interactional Milieu: Implications for Identity Construction and Social Exclusion. Contemporary Voice of Dalit, 10(1), 98-113.

LAMAS, MARTA. (2013). Introducción (pp.9-20). En Lamas, Marta (Comp.) El género. La construcción cultural de la diferencia sexual. México D.F: Porrúa.

LARROSA, JORGE. (2003). Entre las lenguas. Lenguaje y educación después de Babel. Barcelona: Laertes.

LIBRERÍA DE MUJERES DE MILÁN. (1991). No creas tener derechos. Madrid: Horas y Horas. LUKE, CARMEN (1999). Feminismos de la vida cotidiana. Madrid: Morata. 
MACEIRA, LUZ. (2008). El Sueño y la Práctica de Sí: Pedagogía Feminista: Una Propuesta. México D.F: El Colegio de México, A.C., Biblioteca Miguel Cosio Villegas.

MALVANKAR, ALKA. (2019). Students in Goa: Social Lives, Schooling, School Orientations and Aspirations. Social Change, 49(2), 216-231.

MARTÍNEZ, IRENE Y RAMÍREZ, GEMA. (2017). Des-patriarcalizar y Descolonizar la educación. Experiencias para una Formación feminista del profesorado. Revista Internacional de Educación para la Justicia Social (RIEJS), 6(2), 81-95.

MARTÍNEZ, IRENE. (2016). Construcción de una pedagogía feminista para una ciudadanía transformadora y contra-hegemónica. Foro de Educación, 14(20), 129-151.

MOHANTY, CHANDRA. (2008). Bajo los ojos de Occidente: academia feminista y discursos coloniales. En Suarez, Liliana y Hernández, Rosalva (Eds.) Descolonizando el feminismo: teorías y prácticas desde los márgenes (pp.117-164). Madrid: Cátedra.

MOLINA, MARÍA DOLORES. (2011). Investigar la práctica viva de la renovación pedagógica. Revista DUODA: Estudis de la diferència sexual, 40, 111-127.

MOLINA, MERCEDES. (2016). La sociología del sistema de enseñanza de Bourdieu: reflexiones desde América Latina. Cadernos de Pesquisa, 46(162), 942-964.

MONTENEGRO, CATALINA. (2019). La creación artística como proceso reflexivo del SER en femenino: el caso de la escuela chilena. Revista estudios sobre arte actual, nำ7, 113-118.

- (2018). Propuesta de didáctica feminista para la enseñanza escolar de las artes visuales: pensar el espacio escolar de otra manera. Estudios Pedagógicos, XLIV, n³3, 289-302.

MONTOYA, MARÍA MILAGROS. (2008). Enseñar: una experiencia amorosa. Madrid: Sabina.

OCAMPO, JAVIER. (2008). Paulo Freire y la pedagogía del oprimido. Revista historia de la educación latinoamericana, (10), 57-72.

PALMA, IRMA. (2018). Debates abiertos en la coyuntura sobre las instituciones universitarias por las estudiantes del movimiento mayo feminista. Anales de la Universidad de Chile, $\mathrm{n}$-14, 89-107.

PIUSSI, ANA. (2000). Partir de sí: necesidad y deseo. Revista DUODA: Estudis de la diferència sexual, 19, 107-126.

PÉREZ, ANA, NOGUEROLES, MARTA y MÉNDEZ, ÁNGEL. (2017). Una educación feminista para transformar el mundo. Revista Internacional de Educación para la Justicia Social (RIEJS), 6(2), 5-10.

PORTER, ELISABETH. (1999). Mujeres y amistades: Pedagogías de la atención personal y las relaciones. En Luke, Carmen. (Ed.), Feminismos de la vida cotidiana (pp.67-86), Madrid: Morata.

PULEO, RENATA. (2009). El tiempo de la experiencia. En Lelario, Antonietta; Cosentino, Vita y Armellini, Guido (Ed.) Buenas noticias de la escuela (pp.113-118). Madrid: Sabina.

REYES-HOUSHOLDER, CATHERINE Y ROQUE, BEATRIZ. (2019). Chile 2018: desafíos al poder de género desde la calle hasta La Moneda. Revista de ciencia política (Santiago), 39(2), 191-216. 
RICH, ADRIENNE. (1979). Nacida de mujer. La crisis de la maternidad como institución y como experiencia. Barcelona: Noguer.

RICHARD, NELLY. (2018). La insurgencia feminista de mayo 2018. En Zerán, Faride. (Ed.), Mayo Feminista. La rebelión contra el patriarcado (pp.115-126). Santiago: LOM.

RICOEUR, PAUL. (1999). Historia y narratividad. Barcelona: Paidós.

RIVERA, MARÍA MILAGROS. (2012). El amor es el signo. Educar como educan las madres. Madrid: Sabina.

RODRÍGUEZ, TERESA. (2002). La educación, un espacio fundamental femenino en la labor de civilización. En Montoya, María Milagros (Ed.) Escuela y educación ¿Hacia dónde va la libertad femenina? (pp.52-53). Madrid: Horas y horas.

SEOANE,VIVIANA Y SEVERINO, MOIRA. (2019). Género, sexualidades y educación. Intersecciones necesarias para una educación emancipadora. Descentrada, vol.3, nํ1, 1-6.

TIPLER, CAROLINE Y RUSCHER, JANET. (2019). Dehumanizing representations of women: the shaping of hostile sexist attitudes through animalistic metaphors. Journal of Gender Studies, 28(1), 109-118.

TRONCOSO LELYA, FOLLEGATI, LUNA Y STUTZIN, VALENTINA. (2019). Más

allá de una educación no sexista: aportes de pedagogías feministas interseccionales. Pensamiento Educativo, 56(1), 1-15.

TOMÉ, AMPARO Y RAMBLA, XAVIER. (2001). Contra el sexismo. Coeducación y democracia en la escuela. Barcelona: Síntesis/ ICE-UAB.

VALDÉS, TERESA. (2013). Género en la escuela, o la porfiada desigualdad. Revista Docencia, $\mathrm{n}-49,46-61$.

SANTOS, ANTONIA, MEDEL, EVELYNE, SCHUSTER, SOFÍA, ARCE, JAVIERA, ROQUE, BEATRIZ Y MIRANDA LUCIA. (2019). Una mirada al movimiento feminista en Chile del año 2018: hitos, agenda y desafíos. Iberoamericana, 19(72), 223-245.

UNDURRAGA, ROSARIO Y SIMBÜRGER, ELISABETH. (2018). Género y políticas institucionales en universidades chilenas: un desierto con incipientes oasis estatales. En Mora, Claudia, Kottow, Andrea, Osses, Valentina y Ceballos, Marco (Ed.) El género furtivo. La evidencia interdisciplinar del género en el Chile actual. (pp. 239-257). Santiago: LOM

UNIDAD DE EQUIDAD DE GÉNERO. (2015) Educación para la igualdad de género. Plan 2015-2018. Santiago: MINEDUC. Disponible en https://www.mineduc.cl/wp-content/uploads/sites/19/2017/01/CartillaUEG.pdf

VAN MANEN, MAX. (1998). El tacto en la enseñanza. El significado de la sensibilidad pedagógica. Barcelona: Paidós.

VOYER, ANDREA. (2018). Of lads and ear'oles: School, work, and the microsociology of social reproduction. Ethnography, 19(4), 565-576. 